\title{
ОБ ИСПОЛЬЗОВАНИИ ОПТИМАЛЬНОЙ ЛИНЕИНОЙ ФИЛЬТРАЦИИ ДЛЯ РЕШЕНИЯ НЕКОРРЕКТНЫХ ЗАДАЧ
}

\author{
(Представил Я. Эйнасто)
}

\section{1. Постановка задачи}

Рассмотрим интегральное уравнение Фредгольма I рода с разностным ядром

$$
\int_{-\infty}^{\infty} k\left(x-x^{\prime}\right) u\left(x^{\prime}\right) d x^{\prime}=v(x), \quad x \in(-\infty, \infty)
$$

или, более кратко,

$$
K u=v .
$$

Функция $v$ известна, требуется определить $u$. Ядро $k(x)$ предполагается неотрицательным и нормированным к единице

$$
k(x) \geqslant 0, \quad \int_{-\infty}^{\infty} k(x) d x=1 .
$$

Задача (1) часто возникает при дистанционном зондировании атмосферы в оптическом диапазоне спектра. В этом случае $u(x)$ представляет собой истинную яркость атмосферы, $v(x)$ - измеренный оптическим сканирующим устройством сигнал, $x, x^{\prime}-$ пространственную координату, а интегральный оператор $K$ описывает сглаживание прибором измеряемой яркости по полю зрения.

Второй, весьма важной сферой применения уравнения (1) является спектроскопия. В этом случае аргумент $x, x^{\prime}$ представляет длину волны или частоту излучения, интегральный оператор $K$ описывает размывание по спектру измеряемого сигнала $u(x)$, которое происходит в спектральном приборе.

Уравнение (1) допускает также следующую общую интерпретацию: через сглаживающий фильтр $K$ пропускается сигнал $u$, на выходе регистрируется сглаженный сигнал $v$. По данным измерения сглаженного сигнала требуется определить истинный сигнал $u$. Характерным признаком уравнения (1) является то, что в нем измеренную величину $u$ и регистрируемый сигнал $v$ можно интерпретировать как различные состояния одного и того же физического объекта. Если, например, $u$ яркость лимба Земли, то и $v$ можно толковать тоже как яркость лимба (только несколько более сглаженной по сравнению с $u$ ). В частности, если размер поля зрения

$$
\sigma=\left[\int_{-\infty}^{\infty} x^{2} k(x) d x\right]^{1 / 2}
$$

намного меньше масштаба изменчивости изучаемого объекта $X$

$$
\sigma \ll X \sim|u| \frac{d u}{d x} \mid
$$

то сглаживающим воздействием прибора можно пренебречь и полагать $u \approx v$. Необходимость в восстановлении возникает тогда, когда условие (4) не выполнено. 
Уравнение (1) является некорректным и для его решения необходимо применить регуляризацию. Из существующих методов регуляризации наиболее известными являются метод оптимальной линейной фильтрации Винера [ $\left.{ }^{1}\right]$, метод Тихонова $\left.{ }^{2-5}\right]$ и метод наиболее гладкого допустимого ансамбля Турчина $\left[{ }^{6-8}\right]$. Подробное описание этих алгоритмов регуляризованного решения уравнения (1) можно найти в монографии $\left[{ }^{9}\right]$.

В настоящей работе мы предлагаем метод решения уравнения (1), который можно рассматривать как своего рода синтез метода оптимальной линейной фильтрации с методами Тихонова и Турчина. Из метода Винера мы заимствуем общую схему решения и критерий оптимизации при выборе оператора восстановления, из методов Тихонова и Турчина - идею представления восстанавливающего оператора как функцию от малого числа параметров.

Мы будем искать приближенное регуляризованное решение уравнения (1) в виде

$$
u_{\tau}=R_{\tau} v,
$$

где $R_{\tau}-$ зависящий от параметров $\tau=\left\{\tau_{1}, \ldots, \tau_{N}\right\}$ линейный оператор. Параметры $\tau_{i}$ будем определять из условия минимума функционала

$$
E\left\|u_{\tau}-u\right\|^{2},
$$

где $E$ - оператор математического ожидания; $\|\cdot\|-$ норма в $L_{2}(-\infty, \infty)$. Таким образом, восстановленное решение будет наилучшим приближением к неизвестному точному сигналу $u$ в среднеквадратичном смысле. Это наиболее существенное отличие предлагаемого метода от методов Тихонова и Турчина, в которых отыскивается наиболее гладкое из всех возможных при заданном уровне погрешности решение. Настоящая работа является логичсским продолжением работы $\left[{ }^{10}\right]$.

\section{2. Оптимальная линейная фильтрация в сочетании с параметризацией восстанавливаюего оператора}

Для решения задачи удобно использовать преобразование Фурье. Обоз!ачим

$$
\begin{gathered}
\tilde{k}(\omega)=F k(x) \equiv \int e^{i \omega x} k(x) d x, \\
\tilde{u}(\omega)=F u(x), \quad \tilde{v}(\omega)=F v(x), \quad \tilde{u}_{\tau}(\omega)=F u_{\tau}(x) .
\end{gathered}
$$

Тогда преобразование Фурье для уравнения (1) будет

$$
\tilde{v}(\omega)=\widetilde{k}(\omega) \cdot \tilde{u}(\omega) .
$$

Непосредственно использовать это соотношение для определения $u(x)$ не удается, т. к. функция $\tilde{u}(\omega)=\widetilde{v}(\omega) / \widetilde{k}(\omega)$ оказывается при $|\omega| \rightarrow \infty$ расходящейся. Причиной тому является наличие в сигнале $v(x)$ погрешности измерения $\delta(x)$, спектральная плотность которой $\tilde{\delta}(\omega)$ не стремится к нулю при $|\omega| \rightarrow \infty$, что и составляет суть некорректности задачи. В дальнейшем целесообразно выделить в измеренном сигнале явно шумовую компоненту и записать вместо (5)

$$
\tilde{v}(\omega)=\widetilde{k}(\omega) \tilde{u}(\omega)+\tilde{\delta}(\omega) .
$$

Предположим, что $\tilde{u}(\omega)$ и $\tilde{\delta}(\omega)$ являются представителями взаимно некоррелированных статистических ансамблей $\{\tilde{u}\}$ и $\{\tilde{\delta}\}$ с известными первыми и вторыми моментами 


$$
\begin{gathered}
E[\tilde{u}(\omega)]<\infty, \quad E\left[|\tilde{u}(\omega)|^{2}\right]=a^{2}(\omega), \\
E[\tilde{\delta}(\omega)]=0, \quad E\left[|\tilde{\delta}(\omega)|^{2}\right]=\varepsilon^{2}(\omega), \\
E\left[\tilde{\delta}^{*}(\omega) \cdot \tilde{u}(\omega)\right]=0 .
\end{gathered}
$$

В частном случае допускается, что ансамбль $\{\tilde{u}\}$ состоит из одного единственного представителя

$$
E\left[|\tilde{u}(\omega)|^{2}\right]=|\tilde{u}(\omega)|^{2} .
$$

Из (5а) для второго момента функции $\widetilde{v}(\omega)$ получим соотношение

$$
E\left[|\tilde{v}(\omega)|^{2}\right]=|\widetilde{k}(\omega)|^{2} a^{2}(\omega)+\varepsilon^{2}(\omega) .
$$

Регуляризованное решение $\tilde{u}_{\tau}(\omega)$ будем искать в виде

$$
\tilde{u}_{\tau}(\omega)=r(\omega) \tilde{v}(\omega),
$$

где $r(\omega)$ - некоторая функция, которая не зависит от конкретных представителей статистического ансамбля $\tilde{u}(\omega)$ и $\tilde{\delta}(\omega)$. Функцию $r(\omega)$ определим из условия минимальности функционала

$$
Q[r]=\frac{1}{2} E \int\left|\tilde{u}_{\tau}-\tilde{u}\right|^{2} d \omega .
$$

Используя (6), (8) и (9), этот функционал можно привести к виду

$$
Q[r]=\frac{1}{2} \int\left[|1-r \widetilde{k}|^{2} a^{2}+|r|^{2} \varepsilon^{2}\right] d \omega .
$$

Если ни на $r$ и ни на $Q$ не наложены никакие ограничения, то из усливия $\delta Q=0$ получим для $r(\omega)$ формулу Винера

$$
r(\omega)=\frac{k^{*}(\omega)}{k(\omega) k^{*}(\omega)+\varepsilon^{2}(\omega) / a^{2}(\omega)} .
$$

При таком выборе восстанавливающего оператора решение (9) является наилучшим приближением к искомому профилю $\widetilde{u}(\omega)$ в среднеквадратичном смысле. Однако на практике полученное решение, как правило, использовать не удается, т. к. функция $a^{2}(\omega)$ неизвестна или известна недостаточно точно.

Изменим теперь несколько постановку задачи. Будем искать функцию $r(\omega)$ параметрически, предполагая, что

$$
r(\omega)=k^{*}(\omega) \cdot s(\omega, \tau)
$$

где $s(\omega, \tau)$ - известная функция от частоты $\omega$ и параметров $\tau=$ $=\left\{\tau_{1}, \ldots, \tau_{N}\right\}$. Параметры $\tau_{i}$ будем подбирать таким образом, чтобы функционал (10), который теперь можно рассматривать как функцию $\tau$

$$
\begin{gathered}
Q[r(\tau)] \equiv q(\tau)= \\
=\frac{1}{2} \int\left[|1-s(\omega, \tau)| \widetilde{k}(\omega)\left|{ }^{2}\right|^{2} a^{2}(\omega)+|s(\omega, \tau) \widetilde{k}(\omega)|^{2} \varepsilon^{2}(\omega)\right] d \omega,
\end{gathered}
$$

принял минимальное значение. Это приведет к уравнениям

$$
\int \frac{\partial s(\omega, \tau)}{\partial \tau_{i}}\left[\left(s|\widetilde{k}|^{2}-1\right) b^{2}+s|\widetilde{k}|^{2} \varepsilon^{2}\right] d \omega=0, \quad i=1, \ldots, N .
$$

Здесь введено обозначение

$$
b^{2}(\omega)=|k(\omega)|^{2} a^{2}(\omega),
$$


и, кроме того, предполагается, что $s(\omega, \tau)$ является действительно̀ симметричной относительно точки $\omega=0$ функцией (такую структуру функции $s(\omega, \tau)$ подсказывает формула (11)).

Если при заданном $s(\omega, \tau)$ параметры $\tau_{i}$ подобраны так, что уравнения (13) удовлетворены, то решение (9) с восстанавливающим оператором (12) дает (при заданном $s$ ) наилучшее приближение к точному сигналу $\widetilde{u}$ в среднеквадратичном смысле.

В общем случае (13) представляет собой систему нелинейных уравнений относительно неизвестнь:х параметров $\tau_{i}$. Для решения этой системы нужно знать вторые одноточечные моменты $b^{2}(\omega)$ и $\varepsilon^{2}(\omega)$.

Характер этих моментов варьирует от задачи к задаче, поэтому могут возникать самые разнообразные ситуации. Разберем один из наиболее часто встречаемых случаев. Предположим, что

1) случайный ансамбль $\{\tilde{u}\}$ состоит из одной функции $\widetilde{u}(\omega)$ (т. е. имеет место (7));

2) шум и полезный сигнал в спектре измеренной функции $v$ разделяются

$$
\begin{array}{ll}
|\widetilde{k}(\omega) \tilde{u}(\omega)| \gg \varepsilon(\omega), & |\omega|<\Omega, \\
|\widetilde{k}(\omega) \tilde{u}(\omega)| \ll \varepsilon(\omega), & |\omega|>\Omega ;
\end{array}
$$

3) спектральная плотность шума $\varepsilon(\omega)$ является в первом приближении постоянной величиной

$$
\varepsilon(\omega)=\varepsilon_{0}=\text { const. }
$$

4) основной вклад в интегралы (13) даст область $|\omega|<\Omega$ (выполнение этого условия в значительной степени зависит от выбора функции $s(\omega, \tau)$.

Уравнения (13) можно при выполнении предположений 1)-4) заменить на следующую приближенную систему

$$
\int_{-\Omega}^{\Omega} \frac{\partial s}{\partial \tau_{i}}\left(s|\widetilde{k}|^{2}-1\right)|\widetilde{v}|^{2} d \omega+\varepsilon_{0}^{2} \int_{-\infty}^{\infty} s \frac{\partial s}{\partial \tau_{i}}|\widetilde{k}|^{2} d \omega=0, \quad i=1, \ldots, N .
$$

Входящие сюда величины $\widetilde{v}(\omega), \Omega$ и $\varepsilon_{0}$ оцениваются из конкретного единичного эксперимента. Таким образом, при каждом измерении сигнала $v(x)$ получаются различные, оптимальные для данного конкретного нзмерения, значения параметров $\tau_{i}$.

Отметим, что уравнения (16) можно получить из общей системы (13), если относительно случайного ансамбля $\{\tilde{\delta}\}$ сделать предположение (15), а относительно ансамбля $\{\tilde{u}\}-$ предположение

$$
b^{2}(\omega)= \begin{cases}|\tilde{v}(\omega)|^{2}, & |\omega|<\Omega, \\ 0, & |\omega|>\Omega .\end{cases}
$$

Это предположение означает, что мы априори считаем $\{u(x)\}$ гладким ансамблем, в котором отсутствуют высокие частоты в области $|\omega|>\Omega$.

\section{3. Примеры параметризации восстанавливающего оператора}

Рассмотрим некоторые конкретные способы выбора функции $s(\omega, \tau)$ в операторе $(12)$.

А. Пусть $s(\omega, \tau)$ имеет вид

$$
s(\omega, \tau)=1 /\left[|\widetilde{k}(\omega)|^{2}+\sum_{i=0}^{N} \tau_{i} \omega^{2 i}\right] .
$$

Такой ьыбор подсказывается структурой общего оператора 
K такой же функции $s(\omega, \tau)$ приводят в наиболее общем случае метод обобщенной невязки $[4,5]$ и метод наиболее гладкого ансамбля $\left[{ }^{6,7}\right]$.

Подстановка функции (18) в систему (16) приводит к уравнениям

$$
\sum_{j=0}^{N} \tau_{j} \int_{0}^{\Omega} \frac{\omega^{2(i+j)}|\tilde{v}(\omega)|^{2} d \omega}{\left[|\widetilde{k}(\omega)|^{2}+\sum_{p=0}^{N} \tau_{p} \omega^{2 p}\right]^{3}}=\varepsilon_{0}^{2} \int_{0}^{\infty} \frac{\omega^{2 i}|\widetilde{k}(\omega)|^{2} d \omega}{\left[|k(\omega)|^{2}+\sum_{p=0}^{N} \tau_{p} \omega^{2 p}\right]^{3}},
$$

Частный, наиболее простой случай одномерной параметризации получим, если в (18) будем полагать все $\tau_{i}$ равными нулю, за исключением $\tau_{1}=\tau$,

$$
s(\omega, \tau)=1 /\left[|\widetilde{k}(\omega)|^{2}+\tau \omega^{2}\right] .
$$

Для параметра $\tau$ в этом случае имеем уравнение

$$
\tau \int_{0}^{\Omega} \frac{\omega^{4}|\tilde{v}(\omega)|^{2} d \omega}{\left[|\widetilde{k}(\omega)|^{2}+\tau \omega^{2}\right]^{3}}=\varepsilon_{0}^{2} \int_{0}^{\infty} \frac{\omega^{2}|\widetilde{k}(\omega)|^{2} d \omega}{\left[|\widetilde{k}(\omega)|^{2}+\tau \omega^{2}\right]^{3}} .
$$

Здесь справа стоит малый множитель $\varepsilon_{0}^{2}$, в силу чего и решение $\tau$ окажется малым параметром. Однако нельзя полагать $\varepsilon_{0}=0$, так как в этом случае уравнение (21) не будет иметь решения в области $\tau>0$ и оператор $r(\omega, \tau)$ не будет обладать необходимыми свойствами регулярности.

Б. Если функция $\widetilde{k}(\omega)$ стремится к нулю достаточно быстро при $|\omega| \rightarrow \infty$, то $s(\omega, \tau)$ можно разложить в степенной ряд в окрестности точки $\omega=0$. Это приведет к представлению

$$
s(\omega, \tau)=1+\sum_{i=1}^{N} \tau_{i} \omega^{2 i} .
$$

Здесь для регулярности оператора $r=k^{*} s$ необходимо, чтобы выполнялось условие $k^{*} \cdot \omega^{2 N} \rightarrow 0$ при $|\omega| \rightarrow \infty$. Подстановка выражения (22) в систему (16) приводит к линейным уравнениям для определения $\tau_{i}$. Специфической чертой получаемых уравнений является то, что в них можно пренебречь членами, зависящими от параметра $\varepsilon_{0}$ (т. е. при использовании фсрмулы $(22)$ можно в (16) полагать $\left.\varepsilon_{0}=0\right)$, поэтому будем иметь

где

$$
\sum_{j=1}^{N} A_{i+j} \tau_{j}=B_{i}, \quad i=1, \ldots, N
$$

$A_{i}=\int_{0}^{\Omega} \omega^{2 i}|k(\omega)|^{2}|\tilde{v}(\omega)|^{2} d \omega, \quad B_{i}=\int_{0}^{\Omega} \omega^{2 i}|\tilde{v}(\omega)|^{2}\left[1-|\widetilde{k}(\omega)|^{2}\right] d \omega$.

В частности, при $N=1$ получим

$$
\begin{gathered}
s(\omega, \tau)=1+\tau \omega^{2}, \\
\tau=B_{1} / A_{2} .
\end{gathered}
$$

В. Еще один интересный способ параметризации восстанавливающего оператора получим, если вместо (12) используем формулу

$$
r(\omega, \tau)=1+\widetilde{k}(\omega) \sum_{i=1}^{N} \tau_{i} \omega^{2 i} .
$$

Такая параметризация является корректной (не приводит к противоречиям с требованием спиметрии и действительности функции $\widetilde{k} \cdot r)$, если 
$\widetilde{k}(\omega)$ является четной действительной функцией: $\widetilde{k}(\omega)=\widetilde{k}(-\omega)=\widetilde{k}^{*}(\omega)$. Для параметров $\tau_{i}$ получим (при выполнении условий 1)-4) предыдущего раздела и при пренебрежении членами, зависящими от $\varepsilon_{0}$ ) уравнения (23) со свободными членами $B_{i}$, несколько отличающимися от свободных членов в формулах (24) (матричные элементы $A_{i+j}$ остаются без изменения)

$$
B_{i}=\int_{0}^{\Omega} \omega^{2 i}|\tilde{v}(\omega)|^{2}[1-\widetilde{k}(\omega)] d \omega .
$$

Одномерный частный случай параметризации (25) при $N=1$ был подробно разобран в работе $\left[{ }^{10}\right]$. Замечательное свойство оператора (25) заключается в том, что при нем выполняется (в случае оптимального выбора параметров $\tau_{i}$ ) условие

$$
\left\|\tilde{u}_{\tau}-\tilde{u}\right\|=\|r(\tau) \tilde{v}-\tilde{u}\| \leqslant\|r(0) \tilde{v}-\tilde{u}\|=\|\tilde{v}-\tilde{u}\|,
$$

и, таким образом, оператор (25) всегда улучшает измеренный сигнал.

\section{Л И ТЕ Р А Т У Р А}

1. Wiener, $N$. Extrapolation, Interpolation and Smoothing of Stationary Time Series with Engeneering Applications. New York, Wiley, 1949.

2. Phillips, D. L. // J. Assoc. Comput. Mach., 1962, 9, № 1, 84-97.

3. Тихонов А. Н. // ДАН СССР, 1963, 151, № 3, 501-504.

4. Тихонов A. Н., Арсенин В. Я. Методы решения некорректных задач. М., Наука, 1979.

5. Тихонов А. Н., Гончаровский А. В., Степанов В. В., Ягола А. Г. Регуляризующие алгоритмы и апрнорная информация. М., Наука, 1983 .

6. Турчин В. Ф. // Ж. вычисл. мат. и мат. физ., 1967, 7, № 6. 1270-1284.

7. Турчин В. Ф. // Ж. вычисл. мат. и мат. физ., 1968, 8, № 1, 230-238.

8. Турчин В. Ф., Нозик В. З. // Изв. АН СССР. Физ. атмосф. и океана, 1969, V, № $1,29-38$,

9. Верлань А. Ф., Сизиков В. С. Интегральные уравнения: методы, алгоритмы, программы, Киев, Наукова думка, 1986.

10. Рыьым Р. // Изв. АН ЭССР. Физ. Матем., 1989, 38, № 2, 213-221.

Ннститут астрофизики и физики атмосферы Академии наук Эстонской ССР

\section{Поступила в редакцию} $7 /$ IX 1988

R. ROOM

\section{OPTIMAALSE LINEAARSE FILTREERIMISE KASUTAMISEST EBAKORREKTSETE OLESANNETE LAHENDAMISEL}

Ebakorrektse sidumi tüüpi integraalvōrrandi lahendamiseks soovitatakse kasutada Wieneri lineaarse filtreerimise meetodit koos filtreeriva operaatori parameetrilise etteandmisega, kusjuures parameetrid valitakse optimumprintsiibil.

R. ROOM

\section{ON THE USE OF THE OPTIMAL LINEAR FILTERING FOR THE SOLUTION OF THE ILL-POSED PROBLEMS}

For the solution of the ill-posed problem in the form of the Fredholm's first kind convolution-type integral equation $K u=v$, the optimal linear filtering method, combined with the parametric representation of the filtering operator, is proposed. The regularized solution is sought in the form $u_{\tau}=R_{\tau} v$, where $R_{\tau}$ is a given linear filtering operator depending on the parameters $\tau=\left\{\tau_{1}, \ldots, \tau_{N}\right\}$. The parameters $\tau_{i}$ are to be determined from the minimizing criterion for the optimizing functional $E\left\|u_{\tau}-u\right\|^{2}$, where $E$ represents the operator of the mathematical expectation and $\|\cdot\|$ - the norm in space $L_{2}$. The special cases of the operator $R_{\tau}$ are considered when the determination of the parameters $\tau_{i}$ reduces to the solution of the equations the coefficients of which depend on the function $v$ and the operator $K$ only. 